\title{
The Resistance of Mango (Mangifera indica) Cultivars to Tip Dieback Disease in Florida
}

Leandro J. Ramos, Thomas L. Davenport, Robert T. McMillan, Jr., and S. Pablo Lara, University of Florida, Institute of Food and Agricultural Sciences, Tropical Research and Education Center, Homestead 33031

\begin{abstract}
Ramos, L. J., Davenport, T. L., McMillan, R. T., Jr., and Lara, S. P. 1997. The resistance of mango (Mangifera indica) cultivars to tip dieback disease in Florida. Plant Dis. 81:509-514.

The resistance of Mangifera indica to tip dieback disease caused by Botryosphaeria ribis, anamorphic state Fusicoccum sp., was determined on 361 trees of 122 mango cultivars and relatives planted in the mango germ plasm collection at the University of Florida, Tropical Research and Education Center, Homestead. Three trees from each cultivar were evaluated on a 1 to 5 scale ranging from those free of tip dieback to those with extensive branch necrosis. Each cultivar was assigned to one of eight major systematic groups based on race or geographic origin. Resistance to tip dieback disease was not associated with any of the groups. No significant differences in mean disease severity were found among the Indian, Southeast Asian, West Indian, Haden, or Sandersha parts of the Haden-Sandersha Complex, Turpentine types, or a group of unclassified cultivars. Two Mangifera species (M. odorata and M. zeylanica) showed the lowest mean disease rating. Cultivars were also grouped into monoembryonic and polyembryonic types. Although several mango cultivars, mainly found in the monoembryonic group, appeared to be free of the disease, resistance to tip dieback was not associated with embryony. This study provides the first indication that field resistance to tip dieback may be present in some mango cultivars.
\end{abstract}

Additional keywords: adventive and zygotic embryos, Ascomycotina, Botryosphaeria dothidea, genetics

Tip dieback of mango (Mangifera indica L.), frequently included under the broad term mango decline $(11,12,13,18)$, is a prevalent disease that has been affecting mango trees in southern Florida since the late 1970s. The etiology of mango dieback remained unknown for several years. McSorley et al. $(11,12)$ consistently found high populations of the nematode Hemicriconemoides mangiferae Siddigi in soils around old, declining mango trees. The association between $H$. mangiferae and tree condition was, however, not evident in young trees, and declining trees were observed in young groves where very few $H$. mangiferae were present, suggesting that decline of young trees is due to other factors (12). The association of host nutrition with decline was investigated by Schaffer $(18,19)$. He concluded that manganese and iron were limiting elements in affected trees, but the authors (13) did not determine whether the deficiency of these microelements actually caused mango decline.

The pathological nature of tip dieback was revealed for the first time during

Corresponding author: L. J. Ramos
E-mail: ljr@gnv.ifas.ufl.edu

Accepted for publication 4 February 1997.

Publication no. D-1997-0311-05R

(C) 1997 The American Phytopathological Society pathogenicity studies with fungi isolated from symptomatic mango tissues (15). The primary causal organism of the disease was identified and described as Botryosphaeria ribis Grossenbacher \& Duggar anamorphic state (6). The identity of B. ribis was confirmed by B. C. Sutton, International Mycological Institute (IMI), Kew, Surry, England, with a herbarium specimen deposited as IMI 293744. No name has been formally assigned to the anamorph, but according to the current taxonomic concepts, Fusicoccum sp. is the correct generic name for it (E. Punithalingam, International Mycological Institute, personal communication). B. ribis induced severe dieback in up to $83 \%$ of the inoculated plants (15). Several other fungi were occasionally isolated from symptomatic tissues of mango showing dieback symptoms, including Alternaria sp., Colletotrichum sp., Curvularia sp., Fusarium equiseti (Corda) Sacc., Macrophoma sp., Oidium sp., and Stigmina sp. Moreover, Pestalotiopsis sp. and Chalaroptis sp. were isolated from walls of ambrosia beetle larvae bark galleries found in mango trees. In the pathogenicity studies, some of these fungi caused necrosis around inoculation scars, but none of them induced dieback. The avenues of penetration and infection of unwounded stems or twigs by $B$. ribis are unknown (15). Nevertheless, it seems probable that the host can be weakened by these secondary pathogens that cause external lesions and can facilitate the entrance and infection of endophytic fungi. In addition, because symptoms of mango tip dieback develop after long incubation periods, generally 5 to 10 months (15), infection and disease development are often related to environmental stresses suffered during the incubation period (L. J. Ramos and S. P. Lara, unpublished). Diplodia sp., another anamorphic state of Botryosphaeria, induced mild dieback symptoms in up to $17 \%$ of inoculated plants (15). Diplodia sp. has been previously reported in Florida mangoes (21).

$B$. ribis appears to be an aggressive pathogen that invades the vascular system of mango plants. Histopathological studies of symptomatic tissue revealed the production of tyloses in xylem vessels, necrosis of xylem vessels, formation of dark inclusions, and the presence of fungal hyphae (15). These findings suggested that $B$. ribis infection brings about a disjunction of xylem elements with concomitant restriction of water and mineral transport. $B$. ribis was associated with dieback of young stems and limbs in the Hawaiian Islands as early as 1929 (22).

After artificial inoculations of Keitt mango with different fungal isolates, Ploetz et al. (13) established the relationships of several fungi with all or several mango decline symptoms, such as bud necrosis, tip dieback, gummosis, and vascular discoloration. The authors reported a relatively low incidence (10\%) of tip dieback after inoculations with Dothiorella dominicana Petr \& Cif. (synonym: Fusicoccum aesculi Corda; teleomorph: Botryosphaeria dothidea (Moug:Fr.) Ces \& De Not.) and $12 \%$ incidence after inoculations of Lasiodiplodia theobromae (Pat.) Grifford \& Maubl. (synonym: Diplodia natalensis PoleEvans, Diplodia theobromae Pat.; teleomorph: Botryosphaeria rhodina (Cooke) Arx). An even lower incidence (4\%) of tip dieback was obtained after inoculations with Colletotrichum gloeosporioides (Penz.) Penz. \& Sacc. and Alternaria alternata (Fr:Fr.) Keissl.

Ramos et al. (15) determined B. ribis (anamorph: Fusicoccum sp.) and, less commonly, Diplodia sp. as the primary causal organisms of tip dieback; whereas Ploetz et al. (13) reported B. dothidea (anamorph: Fusicoccum aesculi) and Diplodia theobromae as the fungi most commonly causing tip tieback. B. C. Sutton suggested that Fusicoccum aesculi, the 
Table 1. Severity of tip dieback of mango (Mangifera indica) cultivars and relatives planted in the collection at the University of Florida, Tropical Research and Education Center, Homestead

\begin{tabular}{|c|c|c|c|c|c|}
\hline Cultivar & $\begin{array}{c}\text { Embryo } \\
\text { type }^{\mathrm{a}}\end{array}$ & $\begin{array}{l}\text { Disease } \\
\text { rating }\end{array}$ & Cultivar & $\underset{\text { type }}{\text { Embryo }}$ & $\begin{array}{l}\text { Disease } \\
\text { rating }\end{array}$ \\
\hline \multicolumn{6}{|l|}{ 1. Indian types } \\
\hline Ameeri & M & $2.33 \pm 0.22$ & Langra Benarsi & M & $1.00 \pm 0.00$ \\
\hline Amini & M & $3.33 \pm 0.22$ & Mulgoba & M & $3.00 \pm 0.00$ \\
\hline Bennett & M & $1.67 \pm 0.44$ & Paheri & M & $1.67 \pm 0.44$ \\
\hline Bombay Green & M & $2.00 \pm 0.22$ & Rajpuri & M & $1.33 \pm 0.22$ \\
\hline Borsha & M & $1.33 \pm 0.22$ & Safeda Lucknow & M & $3.00 \pm 0.00$ \\
\hline Gola & M & $1.00 \pm 0.00$ & Sandersha & M & $1.00 \pm 0.00$ \\
\hline Joe Welch & M & $2.67 \pm 0.22$ & White Langra & M & $1.00 \pm 0.00$ \\
\hline \multicolumn{6}{|c|}{ 2. Southeast Asia types } \\
\hline Aroemanis CR-1 & $\mathrm{P}$ & $1.67 \pm 0.22$ & Madoe CR-42 & $\mathrm{P}$ & $2.33 \pm 0.22$ \\
\hline Cambodiana & $\mathrm{P}$ & $3.00 \pm 0.38$ & Micongensis & $\mathrm{P}$ & $2.33 \pm 0.22$ \\
\hline Cecil & $\mathrm{P}$ & $2.00 \pm 0.38$ & Mun & $\mathrm{P}$ & $1.33 \pm 0.22$ \\
\hline Duncan & $\mathrm{M}$ & $2.00 \pm 0.38$ & Nam-Dok-Mai & $\mathrm{P}$ & $2.67 \pm 0.38$ \\
\hline Florigon & $\mathrm{P}$ & $3.33 \pm 0.22$ & Okrung & $\mathrm{P}$ & $3.00 \pm 0.38$ \\
\hline Gadoeng & $\mathrm{P}$ & $2.67 \pm 0.79$ & Philippine & $\mathrm{P}$ & $2.33 \pm 0.22$ \\
\hline Golek-CR-1 & $\mathrm{P}$ & $1.33 \pm 0.22$ & Rockdale Saigon & $\mathrm{P}$ & $1.00 \pm 0.00$ \\
\hline Hindi & $\mathrm{P}$ & $2.67 \pm 0.22$ & RosiGold (M13264) & M & $1.67 \pm 0.00$ \\
\hline Honc Cambodiana & $\mathrm{P}$ & $3.00 \pm 0.00$ & Tutehau (PI 99804) & $\mathrm{P}$ & $2.67 \pm 0.58$ \\
\hline James Saigon & $\mathrm{P}$ & $1.33 \pm 0.22$ & & & \\
\hline \multicolumn{6}{|l|}{ 3. West Indian types } \\
\hline Brander & $\mathrm{P}$ & $3.00 \pm 0.22$ & Mammee & $\mathrm{P}$ & $3.00 \pm 0.22$ \\
\hline Julie & M & $3.00 \pm 0.00$ & Manzano & $\mathrm{P}$ & $1.33 \pm 0.22$ \\
\hline Kurashige & $\mathrm{P}$ & $1.00 \pm 0.00$ & Sophie Fry & M & $1.33 \pm 0.22$ \\
\hline Madame Francis & $\mathrm{P}$ & $3.67 \pm 0.22$ & & & \\
\hline \multicolumn{6}{|c|}{ 4. Haden-Sandersha Complex -Haden part } \\
\hline 4 Way Bombay & $\mathrm{M}$ & $1.67 \pm 0.22$ & Hodson & M & $2.67 \pm 0.22$ \\
\hline Adams & M & $2.33 \pm 0.22$ & Irwin & M & $2.67 \pm 0.22$ \\
\hline Jacquelin & M & $2.67 \pm 0.38$ & Irwin sdg \# 2 & M & $2.67 \pm 0.22$ \\
\hline AG Reasoner & M & $1.33 \pm 0.22$ & Joellen & $\mathrm{P}$ & $1.00 \pm 0.00$ \\
\hline Alice & M & $1.00 \pm 0.00$ & Kent & M & $2.00 \pm 0.38$ \\
\hline Bill Herman & M & $2.33 \pm 0.22$ & Lathrop & M & $1.00 \pm 0.00$ \\
\hline Burgner & M & $3.00 \pm 0.00$ & Lippens & M & $3.00 \pm 0.00$ \\
\hline Carrie & M & $2.33 \pm 0.22$ & Lucille & M & $3.33 \pm 0.58$ \\
\hline Cogshall & M & $1.00 \pm 0.00$ & Miami Late & M & $2.67 \pm 0.22$ \\
\hline Earle No. 1 & M & $1.00 \pm 0.00$ & Momi-K & M & $3.33 \pm 0.22$ \\
\hline Edward & M & $1.33 \pm 0.22$ & Monser & M & $2.00 \pm 0.00$ \\
\hline Ed $\times$ Earle- 15 & $\mathrm{P}$ & $2.33 \pm 0.22$ & Ono & $\mathrm{P}$ & $1.33 \pm 0.22$ \\
\hline Ed. $\times$ Kent 14 & M & $1.67 \pm 0.22$ & Pettigrew & M & $1.00 \pm 0.00$ \\
\hline Eldon & M & $1.33 \pm 0.22$ & Pillsbury & $\mathrm{M}$ & $4.00 \pm 0.00$ \\
\hline Fascell & M & $1.33 \pm 0.22$ & Pillsbury A & M & $1.67 \pm 0.22$ \\
\hline Glenn & M & $2.67 \pm 0.58$ & Pope & M & $3.00 \pm 0.00$ \\
\hline Glazier & $\mathrm{M}$ & $3.33 \pm 0.22$ & Pruter & $\mathrm{M}$ & $1.00 \pm 0.00$ \\
\hline Gouvea & M & $3.00 \pm 0.00$ & Reasoner & $\mathrm{P}$ & $2.00 \pm 0.38$ \\
\hline Graham & M & $2.67 \pm 0.22$ & Simmonds & $\mathrm{P}$ & $1.33 \pm 0.22$ \\
\hline Haden & M & $1.67 \pm 0.44$ & Sunset & M & $2.33 \pm 0.22$ \\
\hline Hansen & $\mathrm{M}$ & $1.00 \pm 0.00$ & Vandyk & $\mathrm{M}$ & $2.33 \pm 0.22$ \\
\hline Harris & M & $2.00 \pm 0.00$ & Wallace & M & $2.67 \pm 0.22$ \\
\hline Harris sdg & M & $1.67 \pm 0.22$ & Zill & M & $2.33 \pm 0.20$ \\
\hline \multicolumn{6}{|c|}{ 5. Haden-Sandersha Complex -Sandersha part } \\
\hline Anderson & $\mathrm{M}$ & $2.33 \pm 0.22$ & Parris & M & $1.00 \pm 0.00$ \\
\hline Beverly & M & $2.00 \pm 0.00$ & Parvin & M & $2.00 \pm 0.00$ \\
\hline Brooks & M & $2.33 \pm 0.22$ & Ruby & M & $1.00 \pm 0.00$ \\
\hline Davis Haden & M & $2.00 \pm 0.00$ & Rycheck & M & $2.33 \pm 0.44$ \\
\hline Dixon & M & $2.67 \pm 0.79$ & Sandersha sdg 6-16 & M & $1.33 \pm 0.22$ \\
\hline Early Gold & M & $2.67 \pm 0.22$ & Scuppernong & M & $3.33 \pm 0.22$ \\
\hline Ford & M & $2.00 \pm 0.00$ & Sensation & M & $2.33 \pm 0.22$ \\
\hline Golden Brooks & M & $2.67 \pm 0.22$ & Smith & $\mathrm{M}$ & $1.67 \pm 0.44$ \\
\hline Keenan & M & $1.00 \pm 0.00$ & Springfels & M & $2.33 \pm 0.22$ \\
\hline Keitt & M & $2.00 \pm 0.00$ & Valencia Pride & M & $1.33 \pm 0.22$ \\
\hline Martin & M & $1.00 \pm 0.00$ & Webber & M & $1.33 \pm 0.22$ \\
\hline \multirow{2}{*}{\multicolumn{6}{|c|}{ 6. Turpentine types }} \\
\hline & & & & & \\
\hline Itamaraca & $\mathrm{P}$ & $3.00 \pm 0.38$ & Saber & $\mathrm{P}$ & $2.50 \pm 0.22$ \\
\hline No. 11 & $\mathrm{P}$ & $3.00 \pm 0.65$ & Stringless Peach & $\mathrm{P}$ & $2.67 \pm 0.22$ \\
\hline Peach & $\mathrm{P}$ & $2.00 \pm 0.38$ & Turpentine & $\mathrm{P}$ & $4.00 \pm 0.38$ \\
\hline Red Itamaraca & $\mathrm{P}$ & $2.33 \pm 0.27$ & & & \\
\hline \multicolumn{6}{|c|}{ 7. Nonclassified cultivars } \\
\hline Golden Delight & $\mathrm{M}$ & $2.67 \pm 0.22$ & Ott & M & $2.67 \pm 0.58$ \\
\hline Kensington & & $1.33 \pm 0.22$ & Wally & M & $1.67 \pm 0.22$ \\
\hline \multicolumn{6}{|c|}{ 8. Other Mangifera species } \\
\hline M. odorata & M & $1.67 \pm 0.22$ & M. zeylanica & M & $1.67 \pm 0.44$ \\
\hline
\end{tabular}

\footnotetext{
${ }^{a}$ Embryo types $(\mathrm{M}=$ monoembryonic and $\mathrm{P}=$ Polyembryonic $)$ were randomly distributed.
}

${ }^{\mathrm{b}}$ Mean disease ratings followed by standard errors (ratings 1 to 5: $1=$ free of tip dieback; $5=$ severely damaged plants). No significant differences among eight major race, groups of complexes. GLM, nested design, using Type III MS cultivar (group) as an error term. (df $=7, F$ value $=1.10, P$ $>0.369$ ). There were significant differences in disease response among individual cultivars using MSE as the error term $(\mathrm{df}=121, F$ value $=3.52, P>0.0001)$. The three replicated trees from each cultivar were not distributed at random. Major groups were randomly distributed. anamorphic state of $B$. dothidea causing canker and dieback in Tetragastris panamensiss (Engl.) Kuntze, is morphologically different from the anamorphic state of $B$. ribis, which some authors consider to be a synonym of $B$. dothidea (4). There is disagreement in the literature regarding the delimitation of both the genus and the species within the genus Botryosphaeria (7). Discrepancy in the species identified by Ramos et al. (15) and Ploetz et al. (13) can be explained by their use of different sources for taxonomic identification and confirmation of isolates. The isolates of Ramos et al. (15) were identified and confirmed by B. C. Sutton; whereas the isolates of Ploetz et al. (13) were identified and confirmed by G. I. Johnson (Queensland Department of Plant Industries, Indooroopilly, Australia).

The Tropical Research and Education Center (TREC), University of Florida, is a repository for numerous monembryonic and polyembryonic mango cultivars representing diverse genetic backgrounds from the world's major races and complexes of mango and its relatives. $(14,16)$. Rhodes et al. (16) constructed phenograms, based on cluster analysis, to assign cultivars to specific systematic groups. Grouping was based on 73 vegetative and reproductive characters, with emphasis on fruit, inflorescence, leaf morphology, and embryony. Combined with evidence of the pathological nature of tip dieback, the trees in the collection at TREC provided the opportunity to investigate the possibility that resistance to tip dieback disease was present in some of these systematic groups. The objective of this study, therefore, was to investigate possible sources of field resistance to tip dieback in individual mango cultivars and to determine if field resistance is associated with a systematic group or embryony of the cultivars.

\section{MATERIALS AND METHODS}

Plant material. The mango germ plasm collection at TREC is a repository for 122 cultivars. The trees are approximately 20 years old and are planted in rows spaced $7.6 \mathrm{~m}$ apart in Rockdale soil (Krome very gravelly loam soil). All cultivars were grafted on Turpentine, polyembryonic nucellar rootstocks. To determine if resistance to tip dieback disease was associated with any major systematic group, each cultivar was assigned to one of the following groups according to phenotypic characteristics previously described by Popenoe (14) and Rhodes et al. (16): (1) Indian types with monoembryonic, roundish fruit; (2) Southeast Asia types, mainly from Indochina, Java, Pacific Island, Philippines, South Vietnam, and Thailand, with polyembryonic, oblong, elongated fruit; (3) West Indian types, mainly from Barbados, Jamaica, and Puerto Rico, with intermediate fruit shapes; (4) Haden-Sandersha Complex-Haden part, hybrids from Flor- 
ida and Hawaii with large elliptic-shaped fruit; (5) Haden-Sandersha ComplexSandersha part, hybrids of Florida and Hawaii with large oblong-shaped fruit; (6) Turpentine types, polyembryonics, mainly from Cuba, with small, elongated fruit; (7) nonclassified cultivars; and (8) other Mangifera species (M. odorata Griff. from Southeast Asia and M. zeylanica Hook from Ceylon). The subdivision of the Haden-Sandersha Complex described by Rhodes et al. (16) into Haden part and Sandersha part is somewhat artificial. It was based mainly on Popenoe's (14) division of mangos grown in Florida into three types: Mulgoba, Alphonse, and Sandersha.

The cultivars were also grouped in the present study as either monoembryonic or polyembryonic according to their embryo type to study the influence of embryony on resistance to tip dieback (Table 1). Classification of cultivars by embryonic type was based on published $(1,16)$ and nonpublished (C. W. Campbell, R. J. Knight, and R. J. Schnell, personal communications) data.

Trees were fertilized with 8-3-9 (NPK) three times per year. Iron chelate (Geigy Sequestrene 138) and minor element sprays were applied periodically to soil and foliage, respectively. Benomyl, chlorothalonil, copper fungicides, and high-volume overhead irrigation were periodically applied to the trees.

Disease evaluation. The presence or absence of tip dieback was recorded for 361 trees in the germ plasm collection representing three replicates of most cultivars. The severity of dieback symptoms in twigs and branches on individual trees was rated using a scale from 1 to 5 , where $1=$ trees free of disease; 2 = an early stage of infection characterized by browning of leaf petioles and midveins and presence of distal or marginal leaf blade necrosis in one or two branches; $3=$ the presence of dead leaves, which may remain attached, in the tips of several branches, vascular browning, and evidence of pathogen invasion of vascular tissues, formation of tyloses in xylem vessels, and dark inclusions and fungal hyphae present in stem sections; $4=$ dead leaves and progressive defoliation extending to many larger branches along with increased severity and spread of vascular symptoms; and $5=$ severe dieback that extended to major portions of the tree. The trees were evaluated for progression of disease symptoms in December 1988 to 1990. Because of the progressive nature of

\begin{tabular}{|c|c|c|c|c|c|c|c|c|c|c|c|c|c|c|c|c|}
\hline Rows & 1 & 2 & 3 & 4 & 5 & 6 & 7 & 8 & 9 & 10 & 11 & 12 & 13 & 14 & 15 & 16 \\
\hline 1 & $\begin{array}{c}2 \\
\text { Rosigld } \\
\end{array}$ & $\begin{array}{c}1 \\
\text { Rosigld } \\
\end{array}$ & $\begin{array}{c}2 \\
\text { Rosigld } \\
\end{array}$ & $\begin{array}{c}2 \\
\text { Wally } \\
\end{array}$ & $\begin{array}{c}2 \\
\text { Wally } \\
\end{array}$ & $\begin{array}{c}2 \\
\text { Wally }\end{array}$ & $\begin{array}{c}2 \\
\text { Vandyke } \\
\end{array}$ & $\begin{array}{c}2 \\
\text { Vandyke } \\
\end{array}$ & \begin{tabular}{|c|}
3 \\
Vandyke \\
\end{tabular} & - & - & - & - &. & $\begin{array}{c}3 \\
\text { Irwin } 2 \\
\end{array}$ & $\begin{array}{c}2 \\
\text { Inwin } 2 \\
\end{array}$ \\
\hline 2 & $\begin{array}{c}1 \\
\text { Red Itam. }\end{array}$ & $\begin{array}{c}2 \\
\text { Red Itam } \\
\end{array}$ & Red Itam. & $\begin{array}{c}2 \\
\text { M.Odor. }\end{array}$ & $\begin{array}{c}2 \\
\text { M.Odor. }\end{array}$ & $\begin{array}{c}1 \\
\text { M.Odor. }\end{array}$ & $\begin{array}{c}1 \\
\text { M.Zey. }\end{array}$ & $\begin{array}{c}3 \\
\text { M.Zey. }\end{array}$ & $\begin{array}{c}1 \\
\text { M.Zey. }\end{array}$ & $\begin{array}{c}2 \\
\text { Philip. }\end{array}$ & $\begin{array}{c}3 \\
\text { Philip. }\end{array}$ & $\begin{array}{c}2 \\
\text { Philip. }\end{array}$ & $\begin{array}{c}4 \\
\text { Ott }\end{array}$ & $\begin{array}{c}1 \\
\mathrm{O} t \mathrm{tt} \\
\end{array}$ & $\begin{array}{c}3 \\
\mathrm{Ott} \\
\end{array}$ & $\begin{array}{c}3 \\
\text { Inwin } 2 \\
\end{array}$ \\
\hline 3 & $\begin{array}{c}1 \\
\text { No. } 11 \\
\end{array}$ & $\begin{array}{c}4 \\
\text { No.11 } \\
\end{array}$ & $\begin{array}{c}4 \\
\text { No.11 } \\
\end{array}$ & $\begin{array}{c}2 \\
\text { S. Peach } \\
\end{array}$ & $\begin{array}{c}4 \\
\text { S. Peach }\end{array}$ & \begin{tabular}{c|}
2 \\
s. Peach \\
\end{tabular} & $\begin{array}{c}1 \\
\text { Peach } \\
\end{array}$ & $\begin{array}{c}3 \\
\text { Peach }\end{array}$ & $\begin{array}{c}2 \\
\text { Peach }\end{array}$ & $\begin{array}{c}2 \\
\text { Saber }\end{array}$ & $\begin{array}{c}3 \\
\text { Saber }\end{array}$ & Saber & $\begin{array}{c}3 \\
\text { Itam. }\end{array}$ & $\begin{array}{c}2 \\
\text { Itam. }\end{array}$ & $\begin{array}{c}4 \\
\text { Itam. }\end{array}$ & Turp. \\
\hline 4 & $\begin{array}{c}3 \\
\text { Mamm. }\end{array}$ & $\begin{array}{c}* \\
\text { Mamm. }\end{array}$ & $\begin{array}{c}3 \\
\text { Brander } \\
\end{array}$ & $\begin{array}{c}3 \\
\text { Brander } \\
\end{array}$ & $\begin{array}{c}3 \\
\text { Brander } \\
\end{array}$ & $\begin{array}{c}2 \\
\text { Ono } \\
\end{array}$ & $\begin{array}{c}1 \\
\text { Ono } \\
\end{array}$ & $\begin{array}{c}1 \\
\text { Ono }\end{array}$ & $\begin{array}{c}1 \\
\text { Kurash. }\end{array}$ & $\begin{array}{c}1 \\
\text { Kurash. }\end{array}$ & $\begin{array}{c}1 \\
\text { Kurash. }\end{array}$ & $\begin{array}{c}* \\
\text { Jullie }\end{array}$ & $\begin{array}{c}3 \\
\text { Juliee }\end{array}$ & $\begin{array}{c}3 \\
\text { Jullie }\end{array}$ & $\begin{array}{c}4 \\
\text { Turp. }\end{array}$ & $\begin{array}{c}4 \\
\text { Turp. }\end{array}$ \\
\hline 5 & $\begin{array}{c}3 \\
\text { Mamm. }\end{array}$ & $\begin{array}{c}1 \\
\text { Kensig. } \\
\end{array}$ & $\begin{array}{c}2 \\
\text { Kensig. }\end{array}$ & $\begin{array}{c}1 \\
\text { Kensig. }\end{array}$ & $\begin{array}{c}1 \\
\text { Parris } \\
\end{array}$ & $\begin{array}{c}1 \\
\text { Parris } \\
\end{array}$ & $\begin{array}{c}1 \\
\text { Parris } \\
\end{array}$ & $\begin{array}{c}2 \\
\text { S. Fry } \\
\end{array}$ & $\begin{array}{c}1 \\
\text { S. Fry } \\
\end{array}$ & $\begin{array}{c}1 \\
\text { S. Fry } \\
\end{array}$ & $\begin{array}{c}1 \\
\text { Manzano } \\
\end{array}$ & \begin{tabular}{c|}
1 \\
Manzano \\
\end{tabular} & $\begin{array}{c}2 \\
\text { Manzano } \\
\end{array}$ & \begin{tabular}{|c|}
3 \\
Tuehau \\
\end{tabular} & $\begin{array}{c}4 \\
\text { Tuehau } \\
\end{array}$ & \begin{tabular}{|c|c}
1 \\
Tuehau \\
\end{tabular} \\
\hline 6 & $\begin{array}{c}3 \\
\text { S.Luck. } \\
\end{array}$ & $\begin{array}{c}1 \\
\text { Sander. } \\
\end{array}$ & $\begin{array}{c}1 \\
\text { Sander. }\end{array}$ & $\begin{array}{c}1 \\
\text { Sander. }\end{array}$ & $\begin{array}{c}1 \\
\text { L.Benars } \\
\end{array}$ & \begin{tabular}{c|}
1 \\
L.Benars:
\end{tabular} & $\begin{array}{c}1 \\
\text { L.Benarsi } \\
\end{array}$ & $\begin{array}{c}1 \\
\text { Gola } \\
\end{array}$ & $\begin{array}{c}1 \\
\text { Gola } \\
\end{array}$ & \begin{tabular}{|c|}
1 \\
Gola \\
\end{tabular} & $\begin{array}{c}3 \\
\text { Joe W. }\end{array}$ & $\begin{array}{c}3 \\
\text { Joe } \mathbf{W}\end{array}$ & $\begin{array}{c}2 \\
\text { Joe } W \\
\end{array}$ & $\begin{array}{c}4 \\
\text { M.Fran. }\end{array}$ & $\begin{array}{c}4 \\
\text { M.Fran. } \\
\end{array}$ & $\begin{array}{c}3 \\
\text { M.Fran. } \\
\end{array}$ \\
\hline 7 & $\begin{array}{c}* \\
\text { S.Luck. }\end{array}$ & $\begin{array}{c}3 \\
\text { S.Luck. } \\
\end{array}$ & $\begin{array}{c}4 \\
\text { Amini } \\
\end{array}$ & $\begin{array}{c}3 \\
\text { Amini } \\
\end{array}$ & $\begin{array}{c}3 \\
\text { Amini } \\
\end{array}$ & $\begin{array}{c}1 \\
\text { Borsha } \\
\end{array}$ & $\begin{array}{c}1 \\
\text { Borsha } \\
\end{array}$ & $\begin{array}{c}2 \\
\text { Borsha } \\
\end{array}$ & $\begin{array}{c}3 \\
\text { Mulgoba } \\
\end{array}$ & \begin{tabular}{|c|}
3 \\
Mulgoba \\
\end{tabular} & $\begin{array}{c}3 \\
\text { Mulgoba }\end{array}$ & $\begin{array}{c}1 \\
\text { Paheri } \\
\end{array}$ & $\begin{array}{c}3 \\
\text { Paheri } \\
\end{array}$ & $\begin{array}{c}1 \\
\text { Paheri } \\
\end{array}$ & $\begin{array}{c}1 \\
\text { Rajuri } \\
\end{array}$ & $\begin{array}{c}1 \\
\text { Rajun } \\
\end{array}$ \\
\hline 8 & $\begin{array}{c}2 \\
\text { Cecil } \\
\end{array}$ & $\begin{array}{c}1 \\
\text { Cecil } \\
\end{array}$ & $\begin{array}{c}3 \\
\text { Cecil } \\
\end{array}$ & $\begin{array}{c}2 \\
\text { Bomb.G. }\end{array}$ & $\begin{array}{c}2 \\
\text { Bomb.G. }\end{array}$ & $\begin{array}{c}3 \\
\text { Bomb.G }\end{array}$ & $\begin{array}{c}2 \\
\text { Ameeri } \\
\end{array}$ & $\begin{array}{c}3 \\
\text { Ameeri }\end{array}$ & $\begin{array}{c}2 \\
\text { Ameeri }\end{array}$ & $\begin{array}{c}1 \\
\text { Bennett }\end{array}$ & $\begin{array}{c}3 \\
\text { Bennett } \\
\end{array}$ & \begin{tabular}{c|}
1 \\
Bennett \\
\end{tabular} & $\begin{array}{c}1 \\
\text { W. Lang. }\end{array}$ & $\begin{array}{c}1 \\
\text { W. Lang. }\end{array}$ & $\begin{array}{c}1 \\
\text { W. Lang. }\end{array}$ & $\begin{array}{c}2 \\
\text { Rajuri }\end{array}$ \\
\hline 9 & $\begin{array}{c}3 \\
\text { E. Gold } \\
\end{array}$ & $\begin{array}{c}3 \\
\text { E. Gold } \\
\end{array}$ & $\begin{array}{c}2 \\
\text { E. Gold } \\
\end{array}$ & $\begin{array}{c}1 \\
\text { EdXKent }\end{array}$ & $\begin{array}{c}1 \\
\text { EdXKent }\end{array}$ & $\begin{array}{c}3 \\
\text { EdXKent }\end{array}$ & $\begin{array}{c}4 \\
\text { Oakrong } \\
\end{array}$ & \begin{tabular}{|c|}
2 \\
Oakrong
\end{tabular} & \begin{tabular}{|c|}
3 \\
Oakrong \\
\end{tabular} & $\begin{array}{c}3 \\
\text { H.Camb } \\
\end{array}$ & $\begin{array}{c}3 \\
\text { H.Camb. }\end{array}$ & $\begin{array}{c}3 \\
\text { H.Camb }\end{array}$ & $\begin{array}{c}2 \\
\text { Nam } \\
\end{array}$ & $\begin{array}{c}2 \\
\mathrm{Nam}\end{array}$ & $\begin{array}{c}4 \\
\text { Nam } \\
\end{array}$ & $\begin{array}{c}1 \\
\text { Gadoeng }\end{array}$ \\
\hline 10 & $\begin{array}{c}1 \\
\text { Mun } \\
\end{array}$ & $\begin{array}{c}2 \\
\text { Mun } \\
\end{array}$ & $\begin{array}{c}3 \\
\text { Duncan } \\
\end{array}$ & \begin{tabular}{|c|}
2 \\
Duncan \\
\end{tabular} & $\begin{array}{c}1 \\
\text { Duncan } \\
\end{array}$ & \begin{tabular}{c|}
2 \\
Madoe \\
\end{tabular} & $\begin{array}{c}2 \\
\text { Madoe }\end{array}$ & \begin{tabular}{|c|}
3 \\
Madoe \\
\end{tabular} & \begin{tabular}{|c|}
3 \\
Florigon \\
\end{tabular} & \begin{tabular}{|c|}
4 \\
Florigon \\
\end{tabular} & \begin{tabular}{|c|}
3 \\
Florigon \\
\end{tabular} & $\begin{array}{c}3 \\
\text { Cambod. } \\
\end{array}$ & $\begin{array}{c}2 \\
\text { Cambod. }\end{array}$ & $\begin{array}{c}4 \\
\text { Cambod. } \\
\end{array}$ & $\begin{array}{c}2 \\
\text { Gadoeng } \\
\end{array}$ & $\begin{array}{c}5 \\
\text { Gadoeng } \\
\end{array}$ \\
\hline 11 & $\begin{array}{c}1 \\
\text { Mun }\end{array}$ & $\begin{array}{c}2 \\
H \text { Hindi }\end{array}$ & $\begin{array}{c}3 \\
\text { Hindi } \\
\end{array}$ & \begin{tabular}{|c|}
3 \\
Hindi \\
\end{tabular} & $\begin{array}{c}1 \\
\text { Simmon } \\
\end{array}$ & $\begin{array}{c}1 \\
\text { Simmon } \\
\end{array}$ & $\begin{array}{c}2 \\
\text { Simmon } \\
\end{array}$ & $\begin{array}{c}1 \\
\text { Edward } \\
\end{array}$ & $\begin{array}{c}1 \\
\text { Edward }\end{array}$ & $\begin{array}{c}2 \\
\text { Edward } \\
\end{array}$ & $\begin{array}{c}2 \\
\text { Micong. }\end{array}$ & \begin{tabular}{|c|}
3 \\
Micong. \\
\end{tabular} & $\begin{array}{c}2 \\
\text { Micong. } \\
\end{array}$ & $\begin{array}{c}2 \\
\text { Aroaman } \\
\end{array}$ & \begin{tabular}{|c|}
2 \\
Aroaman \\
\end{tabular} & $\begin{array}{c}1 \\
\text { Aroaman } \\
\end{array}$ \\
\hline 12 & \begin{tabular}{|c|}
2 \\
D. Haden \\
\end{tabular} & $\begin{array}{c}1 \\
\text { R.Saigon }\end{array}$ & $\begin{array}{c}1 \\
\text { R.Saigon }\end{array}$ & $\begin{array}{c}1 \\
\text { R.Saigon } \\
\end{array}$ & $\begin{array}{c}1 \\
\text { J Saigon } \\
\end{array}$ & \begin{tabular}{|c|}
1 \\
J.Saigon \\
\end{tabular} & $\begin{array}{c}2 \\
\text { J. Saigon } \\
\end{array}$ & $\begin{array}{c}1 \\
\text { Glenn } \\
\end{array}$ & $\begin{array}{c}4 \\
\text { Glenn }\end{array}$ & $\begin{array}{c}3 \\
\text { Glenn }\end{array}$ & $\begin{array}{c}2 \\
\text { EdXEd15 }\end{array}$ & $\begin{array}{c}3 \\
\text { EdXEd15 }\end{array}$ & $\begin{array}{c}2 \\
\text { EdXEd15 }\end{array}$ & \begin{tabular}{|c|}
1 \\
Golek \\
\end{tabular} & \begin{tabular}{|c|}
2 \\
Golek
\end{tabular} & $\begin{array}{c}1 \\
\text { Golek } \\
\end{array}$ \\
\hline 13 & \begin{tabular}{|c|}
2 \\
D. Haden \\
\end{tabular} & $\begin{array}{c}2 \\
\text { D. Haden }\end{array}$ & $\begin{array}{c}1 \\
\text { Keenan } \\
\end{array}$ & $\begin{array}{c}1 \\
\text { Keenan } \\
\end{array}$ & $\begin{array}{c}1 \\
\text { Keenan } \\
\end{array}$ & \begin{tabular}{|c|}
1 \\
Dixon \\
\end{tabular} & $\begin{array}{c}2 \\
\text { Dixon } \\
\end{array}$ & $\begin{array}{c}5 \\
\text { Dixon } \\
\end{array}$ & $\begin{array}{c}1 \\
\text { Martin } \\
\end{array}$ & $\begin{array}{c}1 \\
\text { Martin } \\
\end{array}$ & $\begin{array}{c}1 \\
\text { Martin } \\
\end{array}$ & \begin{tabular}{|c|}
2 \\
Brooks \\
\end{tabular} & $\begin{array}{c}2 \\
\text { Brooks } \\
\end{array}$ & $\begin{array}{c}3 \\
\text { Brooks } \\
\end{array}$ & $\begin{array}{c}4 \\
\text { Osteen }\end{array}$ & $\begin{array}{c}4 \\
\text { Osteen } \\
\end{array}$ \\
\hline 14 & $\begin{array}{c}3 \\
\text { Ryechk } \\
\end{array}$ & $\begin{array}{c}3 \\
\text { Ryechk }\end{array}$ & $\begin{array}{c}1 \\
\text { Ryechk } \\
\end{array}$ & $\begin{array}{c}1 \\
\operatorname{San} .6 \\
\end{array}$ & $\begin{array}{c}1 \\
\text { San. } 6 \\
\end{array}$ & $\begin{array}{c}2 \\
\text { San. } 6 \\
\end{array}$ & $\begin{array}{c}3 \\
\text { G.Brooks }\end{array}$ & $\begin{array}{c}3 \\
\text { G.Brooks } \\
\end{array}$ & $\begin{array}{c}2 \\
\text { G.Brooks } \\
\end{array}$ & $\begin{array}{c}1 \\
\text { Webber } \\
\end{array}$ & $\begin{array}{c}2 \\
\text { Webber }\end{array}$ & $\begin{array}{c}1 \\
\text { Webber }\end{array}$ & $\begin{array}{c}2 \\
\text { Panin } \\
\end{array}$ & $\begin{array}{c}2 \\
\text { Parvin } \\
\end{array}$ & $\begin{array}{c}2 \\
\text { Parin } \\
\end{array}$ & $\begin{array}{c}4 \\
\text { Osteen } \\
\end{array}$ \\
\hline 15 & $\begin{array}{c}1 \\
\text { Smith } \\
\end{array}$ & $\begin{array}{c}3 \\
\text { Smith } \\
\end{array}$ & $\begin{array}{c}1 \\
\text { Smith } \\
\end{array}$ & $\begin{array}{c}2 \\
\text { V. Pride } \\
\end{array}$ & $\begin{array}{c}1 \\
\text { V. Pride } \\
\end{array}$ & $\begin{array}{c}1 \\
\text { V.Pride } \\
\end{array}$ & $\begin{array}{c}2 \\
\text { Keitt } \\
\end{array}$ & $\begin{array}{c}2 \\
\text { Keitt } \\
\end{array}$ & \begin{tabular}{|c|}
2 \\
Keitt \\
\end{tabular} & \begin{tabular}{|c|}
2 \\
Beverly \\
\end{tabular} & \begin{tabular}{|c|c}
2 \\
Beverly
\end{tabular} & \begin{tabular}{|c|}
2 \\
Beverly \\
\end{tabular} & $\begin{array}{c}2 \\
\text { Sensa. }\end{array}$ & $\begin{array}{c}3 \\
\text { Sens. } \\
\end{array}$ & $\begin{array}{c}2 \\
\text { Sensa. } \\
\end{array}$ & $\begin{array}{c}2 \\
\text { Ford } \\
\end{array}$ \\
\hline 16 & $\begin{array}{c}2 \\
\text { Jacq. }\end{array}$ & $\begin{array}{c}2 \\
\text { Jacg. }\end{array}$ & $\begin{array}{c}3 \\
\text { Andersn } \\
\end{array}$ & \begin{tabular}{|c|}
2 \\
Andersn \\
\end{tabular} & \begin{tabular}{|c|}
2 \\
Andersn \\
\end{tabular} & $\begin{array}{c}1 \\
\text { Ruby } \\
\end{array}$ & $\begin{array}{c}1 \\
\text { Ruby } \\
\end{array}$ & $\begin{array}{c}1 \\
\text { Ruby } \\
\end{array}$ & $\begin{array}{c} \\
\text { Spring. }\end{array}$ & $\begin{array}{c}2 \\
\text { Spring. } \\
\end{array}$ & $\begin{array}{c}2 \\
\text { Spring. } \\
\end{array}$ & $\begin{array}{c}3 \\
\text { Scupp. } \\
\end{array}$ & $\begin{array}{c}3 \\
\text { Scupp. }\end{array}$ & $\begin{array}{c}4 \\
\text { Scupp. }\end{array}$ & $\begin{array}{c}2 \\
\text { Ford } \\
\end{array}$ & $\begin{array}{c}2 \\
\text { Ford } \\
\end{array}$ \\
\hline 17 & $\begin{array}{c}4 \\
\text { Jacq. }\end{array}$ & $\begin{array}{c}3 \\
\text { Haden } \\
\end{array}$ & $\begin{array}{c}1 \\
\text { Haden } \\
\end{array}$ & $\begin{array}{c}1 \\
\text { Haden } \\
\end{array}$ & $\begin{array}{c}2 \\
\text { Fascell } \\
\end{array}$ & $\begin{array}{c}1 \\
\text { Fascell } \\
\end{array}$ & $\begin{array}{c}1 \\
\text { Fascell }\end{array}$ & $\begin{array}{c}1 \\
\text { Kent } \\
\end{array}$ & $\begin{array}{c}2 \\
\text { Kent } \\
\end{array}$ & $\begin{array}{c}3 \\
\text { Kent }\end{array}$ & $\begin{array}{c}1 \\
\text { Pittigr. } \\
\end{array}$ & $\begin{array}{c}1 \\
\text { Pittigr. }\end{array}$ & $\begin{array}{c}1 \\
\text { Pittigr. } \\
\end{array}$ & $\begin{array}{c}1 \\
\text { Eldon } \\
\end{array}$ & $\begin{array}{c}1 \\
\text { Eldon } \\
\end{array}$ & $\begin{array}{c}2 \\
\text { Eicon } \\
\end{array}$ \\
\hline 18 & $\begin{array}{c}2 \\
\text { Harris S. }\end{array}$ & $\begin{array}{c}1 \\
\text { Lathrop } \\
\end{array}$ & $\begin{array}{c}1 \\
\text { Lathrop } \\
\end{array}$ & $\begin{array}{c}1 \\
\text { Lathrop }\end{array}$ & $\begin{array}{c}1 \\
\text { Earle } 1 \\
\end{array}$ & $\begin{array}{c}1 \\
\text { Earle } 1 \\
\end{array}$ & $\begin{array}{c}1 \\
\text { Earle1 } \\
\end{array}$ & $\begin{array}{c}1 \\
\text { Pruter } \\
\end{array}$ & $\begin{array}{c}1 \\
\text { Pruter } \\
\end{array}$ & $\begin{array}{c}1 \\
\text { Pruter } \\
\end{array}$ & $\begin{array}{c}3 \\
\text { Irwin }\end{array}$ & $\begin{array}{c}2 \\
\text { Irwin }\end{array}$ & $\begin{array}{c}3 \\
\text { Irwin }\end{array}$ & $\begin{array}{c}4 \\
\text { Glazier } \\
\end{array}$ & $\begin{array}{c}3 \\
\text { Glazier }\end{array}$ & $\begin{array}{c}3 \\
\text { Glazier } \\
\end{array}$ \\
\hline 19 & $\begin{array}{c}1 \\
\text { Harris S. }\end{array}$ & $\begin{array}{c}2 \\
\text { Harris S. }\end{array}$ & $\begin{array}{c}1 \\
\text { Cogshall } \\
\end{array}$ & $\begin{array}{c}1 \\
\text { Cogshall } \\
\end{array}$ & $\begin{array}{c}1 \\
\text { Cogshall } \\
\end{array}$ & $\begin{array}{c}2 \\
\text { zill } \\
\end{array}$ & $\begin{array}{c}2 \\
\text { Zill } \\
\end{array}$ & $\begin{array}{c}3 \\
\text { Zill } \\
\end{array}$ & $\begin{array}{l}* \\
- \\
\end{array}$ & $\begin{array}{l}* \\
- \\
\end{array}$ & $\begin{array}{l}* \\
- \\
\end{array}$ & $\begin{array}{c}3 \\
\text { Reason. }\end{array}$ & $\begin{array}{c}1 \\
\text { Reason. }\end{array}$ & $\begin{array}{c}2 \\
\text { Reason. }\end{array}$ & $\begin{array}{c}2 \\
\text { Camie } \\
\end{array}$ & $\begin{array}{c}2 \\
\text { Camie } \\
\end{array}$ \\
\hline 20 & $\begin{array}{c}1 \\
\text { Alice } \\
\end{array}$ & $\begin{array}{c}1 \\
\text { Alice } \\
\end{array}$ & $\begin{array}{c}1 \\
\text { Alice } \\
\end{array}$ & $\begin{array}{c}2 \\
\text { Pills.A } \\
\end{array}$ & $\begin{array}{c}1 \\
\text { Pills.A } \\
\end{array}$ & $\begin{array}{c}2 \\
\text { Pills.A } \\
\end{array}$ & $\begin{array}{c}4 \\
\text { Pills. } \\
\end{array}$ & $\begin{array}{c}4 \\
\text { Pills. } \\
\end{array}$ & $\begin{array}{c}4 \\
\text { Pills. } \\
\end{array}$ & $\begin{array}{c}3 \\
\text { Gouvea } \\
\end{array}$ & $\begin{array}{c}3 \\
\text { Gouvea } \\
\end{array}$ & $\begin{array}{c}3 \\
\text { Gouvea } \\
\end{array}$ & $\begin{array}{c}3 \\
\text { Momik } \\
\end{array}$ & $\begin{array}{c}4 \\
\text { Momi K. } \\
\end{array}$ & $\begin{array}{c}3 \\
\text { Momi K. } \\
\end{array}$ & $\begin{array}{c}3 \\
\text { Carrie } \\
\end{array}$ \\
\hline 21 & $\begin{array}{c}2 \\
\text { Monser } \\
\end{array}$ & $\begin{array}{c}2 \\
\text { Monser } \\
\end{array}$ & $\begin{array}{c}2 \\
\text { Manser } \\
\end{array}$ & $\begin{array}{c}1 \\
\text { Joeflen } \\
\end{array}$ & $\begin{array}{c}1 \\
\text { Joellen } \\
\end{array}$ & $\begin{array}{c}1 \\
\text { Joellen } \\
\end{array}$ & $\begin{array}{c}1 \\
4 \text { Way } \\
\end{array}$ & $\begin{array}{c}2 \\
4 \text { Way } \\
\end{array}$ & $\begin{array}{c}2 \\
4 \text { Way } \\
\end{array}$ & $\begin{array}{c}2 \\
\text { Harris } \\
\end{array}$ & $\begin{array}{c}2 \\
\text { Harris } \\
\end{array}$ & $\begin{array}{c}2 \\
\text { Hanris } \\
\end{array}$ & $\begin{array}{c}3 \\
\text { Burgner }\end{array}$ & $\begin{array}{c}3 \\
\text { Burgner } \\
\end{array}$ & $\begin{array}{c}3 \\
\text { Burgner } \\
\end{array}$ & $\begin{array}{c}2 \\
\text { A.Reas } \\
\end{array}$ \\
\hline 22 & $\begin{array}{c}2 \\
\text { Sunset } \\
\end{array}$ & $\begin{array}{c}3 \\
\text { Sunset } \\
\end{array}$ & $\begin{array}{c}5 \\
\text { Lucille } \\
\end{array}$ & $\begin{array}{c}3 \\
\text { Lucille } \\
\end{array}$ & $\begin{array}{c}2 \\
\text { Lucille } \\
\end{array}$ & $\begin{array}{c}3 \\
\text { M. Late } \\
\end{array}$ & $\begin{array}{c}2 \\
\text { M. Late } \\
\end{array}$ & $\begin{array}{l}3 \\
\text { M. Late } \\
\end{array}$ & $\begin{array}{c}3 \\
\text { Adams } \\
\end{array}$ & $\begin{array}{c}2 \\
\text { Adams } \\
\end{array}$ & $\begin{array}{c}2 \\
\text { Adams } \\
\end{array}$ & $\begin{array}{c}3 \\
\text { Pope } \\
\end{array}$ & $\begin{array}{c}3 \\
\text { Pope } \\
\end{array}$ & $\begin{array}{c}3 \\
\text { Pope } \\
\end{array}$ & $\begin{array}{c}1 \\
\text { A.Reas. }\end{array}$ & $\begin{array}{c}1 \\
\text { A.Reas. } \\
\end{array}$ \\
\hline 23 & $\begin{array}{c}2 \\
\text { Sunset } \\
\end{array}$ & $\begin{array}{c}3 \\
\text { Lippens } \\
\end{array}$ & $\begin{array}{c}3 \\
\text { Lippens }\end{array}$ & $\begin{array}{c}3 \\
\text { Lippens }\end{array}$ & $\begin{array}{c}1 \\
\text { Hansen }\end{array}$ & $\begin{array}{c}1 \\
\text { Hansen }\end{array}$ & $\begin{array}{c}1 \\
\text { Hansen }\end{array}$ & $\begin{array}{c}2 \\
\text { B.Herm. }\end{array}$ & $\begin{array}{c}2 \\
\text { 8.Herm. }\end{array}$ & $\begin{array}{c}3 \\
\text { B.Herm. }\end{array}$ & $\begin{array}{c}3 \\
\text { Wallace }\end{array}$ & $\begin{array}{c}2 \\
\text { Wallace }\end{array}$ & $\begin{array}{c}3 \\
\text { Wallace }\end{array}$ & $\begin{array}{c}3 \\
\text { Hodson }\end{array}$ & $\begin{array}{c}2 \\
\text { Hodson }\end{array}$ & $\begin{array}{c}3 \\
\text { Hodson }\end{array}$ \\
\hline
\end{tabular}

Fig. 1. Disease ratings and arrangement of mango cultivars in germ plasm collection located at the University of Florida, Tropical Research and Education Center. All indicated trees were included in the study. Top of figure is orientated to north. 
the disease in affected trees, symptom ratings reported here are those observed only in 1990, the most severe of the 3 years of observation.

Isolation and identification. Isolation procedures were slightly modified from those previously described (15). Tissues from trees displaying tip dieback symptoms were collected, surface-sterilized with $1 \%$ sodium hypochlorite for $3 \mathrm{~min}$, and rinsed three times with sterile distilled water. Bark was removed, and sections from the vascular tissue were dissected with a sterile scalpel and placed on potato dextrose agar (PDA) or malt agar. Streptomycin sulfate was added to the first medium to prevent bacterial growth.

B. ribis anamorph Fusicoccum sp. was identified in the laboratory by its colony morphology, rapid growth rate, dark mycelium, and the formation of chlamydospores and immature conidial initials as previously described (15). Affected tissues produced stromatic pycnidia, which yielded hyaline, one-celled pycnidiospores. (15). When possible given the large number of samples, the isolates were grown side-by-side with our IMI 293744.

Statistical analysis. The plot design was dictated by the previously established arrangement of cultivars in the germ plasm collection. The tree replicates of each cultivar were planted adjacent to each other in the collection (Fig. 1). The major systematic groups or embryonic types were randomly distributed throughout the collection. The General Linear Models procedure (GLM), nested analysis of variance (SAS Institute, Cary, NC), was used to compare disease responses among the major mango systematic groups and between the embryonic types. The type III $F$ value, using MS for cultivar (nested with group), was used as an error term to compare disease responses among the major systematic groups. Similarly, the Type III $F$ value, with MS representing cultivar (nested with embryo), was used as an error term to compare the two embryonic types. The $F$ test with MSE as the error term was used to determine variability of disease responses among cultivars within major systematic groups and embryonic types. Embryonic type was regarded as a fixed effect; whereas cultivar was treated as a random effect (SAS).

The assumption of a homogeneous distribution of disease scores throughout the field was tested by fitting a second-degree polynomial regression model to the scores, based on row and column coordinates, with row and column indices as predictors. An $F$ test was used to compare this model with one with only an intercept term. The disease scores were modeled as a function of row, row ${ }^{2}$, column, column ${ }^{2}$, and a

Table 2. Severity of tip dieback disease of major groups, races or complexes of mango (Mangifera indica L.) and relatives in the germ plasm collection at the University of Florida, Tropical Research and Education Center, Homestead

\begin{tabular}{|c|c|c|c|c|c|}
\hline \multirow[b]{2}{*}{ Major group } & \multirow{2}{*}{$\begin{array}{c}\text { No. of } \\
\text { trees }\end{array}$} & \multirow{2}{*}{$\begin{array}{c}\text { No. of } \\
\text { cultivars }\end{array}$} & \multicolumn{2}{|c|}{ Mean disease ratinga } & \multirow{2}{*}{$\begin{array}{l}\text { \% Disease } \\
\text { free cvs }\end{array}$} \\
\hline & & & Total cvs & $\overline{\text { Diseased cvs }}$ & \\
\hline Indian types & 41 & 14 & $1.85 \pm 0.15$ & 2.210 .17 & 29 \\
\hline Southeast Asia types & 57 & 19 & $2.23 \pm 0.13$ & 2.300 .13 & 5 \\
\hline West-Indian types & 19 & 7 & $2.26 \pm 0.25$ & 2.500 .25 & 14 \\
\hline Haden-Sandersha-Haden part & 138 & 46 & $2.09 \pm 0.08$ & 2.320 .08 & 17 \\
\hline Haden-Sandersha-Sandersha part & 69 & 23 & $2.03 \pm 0.11$ & 2.250 .12 & 17 \\
\hline Turpentine types & 19 & 7 & $2.74 \pm 0.24$ & 2.740 .24 & 0 \\
\hline Nonclassified cultivars & 12 & 4 & $2.08 \pm 0.29$ & 2.080 .29 & 0 \\
\hline \multicolumn{6}{|l|}{ Mangifera spp. } \\
\hline (M. odorata and M. zeylanica) & 6 & 2 & $1.67 \pm 0.33$ & 1.670 .33 & 0 \\
\hline
\end{tabular}

a Mean disease ratings 1 to 5: $1=$ free of tip dieback, $5=$ severely damaged plants. GLM, nested. No significant differences were found among major races or groups of cultivars. Test hypothesis using the type III MS for cultivar (group) as an error term ( $\mathrm{df}=7, F$ value $1.10, P>0.369)$. Groups or races were randomly distributed. The three replicated trees from each cultivar were not distributed at random.

Table 3. Severity of tip dieback disease as related to the embryony of cultivars in the germ plasm collection at the University of Florida, Tropical Research and Education Center, Homestead

\begin{tabular}{|c|c|c|c|c|c|c|c|}
\hline \multirow[b]{2}{*}{ Embryo type } & \multicolumn{4}{|c|}{ Total population studied } & \multicolumn{3}{|c|}{$\begin{array}{c}\text { Population of diseased } \\
\text { cultivars }^{b}\end{array}$} \\
\hline & $\begin{array}{l}\text { No. of } \\
\text { cultivars }\end{array}$ & $\begin{array}{l}\text { No. of } \\
\text { trees }\end{array}$ & $\begin{array}{l}\text { Disease } \\
\text { rating }\end{array}$ & $\begin{array}{l}\% \text { disease } \\
\text { free cvs }\end{array}$ & $\begin{array}{l}\text { No. } \\
\text { of cvs }\end{array}$ & $\begin{array}{l}\text { No. of } \\
\text { trees }\end{array}$ & $\begin{array}{l}\text { Disease } \\
\text { rating }\end{array}$ \\
\hline Monoembryonic & 86 & 256 & $2.06 \pm 0.06$ & 17 & 32 & 214 & $2.28 \pm 0.06$ \\
\hline Polyembryonic & 36 & 105 & $2.23 \pm 0.10$ & 8 & 33 & 93 & $2.34 \pm 0.10$ \\
\hline
\end{tabular}

row.column interaction term. A finding of significance in any one of these terms indicated nonrandom distribution (SAS).

\section{RESULTS}

The mango cultivars used in this study were organized into eight major groups, as previously stated. Table 1 lists the cultivars assigned to each group and includes the embryony as well as the disease ratings for each cultivar. The lowest mean disease rating was found in the group formed by other Mangifera spp. (M. odorata and M. zeylanica), and the highest was found in the Turpentine types; however, when these results were compared with the other groups, no significant differences in severity of tip dieback disease were found (Tables 1 and 2) ( $\mathrm{df}=7, F$ value $1.10, P>$ 0.3690). To learn the effect of those cultivars free of the disease in the disease ratings of each major group, disease-free cultivars were excluded from the population. No significant differences were found among cultivars (df $=7, F=0.88, P>$ 0.5262 ). There were, however, significant differences in disease severity among individual cultivars $(\mathrm{df}=121, F$ value $=3.52$, $P>0.0001)$.

A number of disease-free mango cultivars were found. Of the Indian monoembryonic group of cultivars, four out of 14 (29\%) were disease free. Eight out of 46 in the Haden part (17\%) and seven out of 23 in the Sandersha part $(17 \%)$, both of the mainly monoembryonic group of HadenSandersha Complex, were free of the disease (Tables 1 and 2). The West Indian group, composed of monoembryonic and polyembryonic cultivars, displayed one out of seven (14\%) disease-free cultivars. Only two out of $19(10 \%)$ of the cultivars in the polyembryonic Southeast Asia group and none of the cultivars in the polyembryonic Turpentine group were disease free.

Because most of the disease-free cultivars were found to be monoembryonic, we tested the possibility that disease resistance was associated with embryony. Although $17 \%$ of the monoembryonic cultivars were free of the disease and only $8 \%$ were disease free in the polyembryonic population, no significant differences in mean tip dieback disease ratings were found between the two populations (Table 3). The mean tip dieback disease ratings of the monoembryonic and polyembryonic cultivars were 2.06 and 2.23 , respectively ( $\mathrm{df}=1, F$ value $=1.40, P>0.2388$ ). We also considered the possibility that Haden-Sandersha Complex cultivars, which are composed of Florida and Hawaiian hybrids of Indian and Southeast Asian origin, may display intermediate characteristics. When these groups were removed from the data set, the means of dieback disease ratings were comparable to the previous analysis (1.99 for monoembryonic and 2.28 for polyembryonic cultivars). The analysis of variance of the $F$ test reduced the $P$ value; however, 
differences in disease responses were not significant $(\mathrm{df}=1, F$ value $=2.37, P$ > $0.129)$. There was significant variability in disease responses among the cultivars within each embryo type (df $=121, F=$ $3.47, P>0.0001)$. To determine the effects of the cultivars that were free of the disease on the mean, the 17 cultivars free of disease (14 in the monoembryonic group and three in the polyembryonic group) were excluded from the computation (Table 3). The mean disease ratings were 2.28 and 2.34 for the monoembryonic and polyembryonic populations, respectively. No significant differences were found $(\mathrm{df}=$ $104, F=0.42, P>0.5167)$.

B. ribis anamorph Fusicoccum sp. was the only fungus found in high frequency in symptomatic tissues of diseased trees sampled in all areas of the orchard (data not shown). The incidence of recovery of $B$. ribis was $42 \%$ of the total number of symptomatic trees examined. This frequency of recovery was much higher than that of any other isolated fungus. B. ribis was not found in asymptomatic tissues. Diplodia sp. was also isolated from diseased trees but in much lower frequency, as were other previously reported secondary fungi (15).

The disease scores appeared to be uniformly distributed throughout the field (Fig. 1). High and low disease scores were found in most areas. The assumption of a homogenous distribution of the scores was not supported after examination of the second-degree polynomial regression model to the scores based on row and column coordinates. This second-degree model fit significantly better $(F=5.10$; model $\mathrm{df}=$ 5, error df $=349 ; P>0.0002)$ than did the model with only an intercept term $(P>$ $0.0002)$, indicating that similar disease scores were not uniformly distributed across the field. The $F$ test indicated significance between rows $(P>0.025)$ and no significance between columns $(P>0.146)$ or in the interaction between rows and columns $(P>0.573)$.

\section{DISCUSSION}

The primary objective of this study was to investigate the possibility that disease resistance to mango tip dieback is associated with one or more major mango races, groups, or complexes described in earlier systematic studies $(14,16)$. No attempt was made to group the cultivars according to other comparable phylogenetic studies $(2,5,20)$ because they did not include the cultivars or Mangifera species observed in the present research. A consistently high incidence of disease was found throughout the population of trees. B. ribis was isolated from trees in all sections. A small number of individual trees among the three replicate trees of some cultivars escaped infection. Although no significant differences in the mean disease ratings of tip dieback disease were found among the systematic groups of mango cultivars or relatives, there were distinct differences in the proportion of cultivars found to be disease free in the various groups. Most of the cultivars found to be free of disease occurred in groups dominated by monoembryonic types (Table 1). The exclusively monoembryonic Indian group displayed the highest proportion of disease-free cultivars; whereas the groups composed exclusively of polyembryonic types, such as the Southeast Asia and Turpentine groups, displayed the lowest proportion (Table 2).

Although the proportion of disease-free cultivars in the monoembryonic types was twice as high as that of the polyembryonic types, no difference in the mean disease rating was found between the two types. High disease ratings found in susceptible monoembryonic cultivars offsetting the higher proportion of disease-free cultivars found in that type may account for the similar means.

It is plausible that a greater proportion of the monoembryonic population of cultivars was resistant to tip dieback disease than were the polyembryonic types, because of the greater probability of resistance introduction in the former through sexual reproduction $(3,8)$. Zygotic embryos of monoembryonic cultivars would theoretically have the greater evolutionary advantage in developing resistance to the pathogen than would the adventive, asexual embryos in seeds of polyembryonic types. The latter are derived from the nucellar inner integument of the ovule $(10,17)$. Although Sturrock (23) considered polyembryony to be the more primitive character, and some reversion in embryony has been observed (23), Kostermans and Bompard (9) listed only three out of 69 Mangifera species (M. indica, M. casturi, and $M$. laurina) where polyembryony occurs. It is thus difficult to accept Sturrocks original contention. Rhodes et al. (16) found good separation of phenotypic characters between monoembryonic and polyembryonic cultivars. There was substantial variability in disease responses among the cultivars within each embryony type.

One source of variability of disease rating in the major mango races may be derived from the large number of mango hybrids of intermediate nature within the Florida and Hawaii Haden-Sandersha Complex. Many of these mango cultivars have been developed from seedlings of natural crosses between the diverse monoembryonic and polyembryonic types whose centers of origin are India and Southeast Asia, respectively. As observed by Rhodes et al. (16) in their systematic phylogenetic work, the inclusion of these hybrids exerted an intermediate effect on the clustering of major groups. Removal of the Haden-Sandersha Complex cultivars from the data set decreased the variability displayed by the two embryonic types but was not sufficient to result in a statistical difference between them. There was also a lack of significant difference between the monoembryonic Indian and polyembryonic Southeast Asia groups (Table 2).

B. ribis was consistently isolated from symptomatic tissues of diseased trees located in most areas of the orchard (data not shown). The disease scores were apparently uniformly distributed throughout (Fig. 1), and high and low disease scores were found in most areas, suggesting that the fungus inoculum was available in all sections of the orchard. Nevertheless, the assumption of a homogeneous distribution of disease scores was not supported by the statistical test in rows, and the hypothesis of uniformity was rejected. Significant differences were found among rows, but no significant differences were found among columns or in the interaction of rows and columns. These findings suggest that differences attributed to cultivars may actually be influenced by conditions in the row, but most likely suggest that indices of the rows may be influenced by the clustering of the three replications of each mango cultivar in the rows. The disadvantages of the preset conditions with the three nonrandom replications of each cultivar within rows were mentioned previously.

The study presented here provides the first indication that field resistance to tip dieback disease may be present in some mango cultivars. These results are valuable for development of cultivars and future research; efforts should include the evaluation of those cultivars showing field resistance using controlled conditions.

\section{ACKNOWLEDGMENTS}

We thank C. W. Campbell, R. J. Knight, Jr., and R. J. Schnell for providing information on the embryony of several mango cultivars. We also thank J. M. Harrison and F. G. Martin for their statistical advice and J. T. O'Neal for editorial suggestions. University of Florida, Agricultural Experiment Station Journal Series No. R-03849.

\section{LITERATURE CITED}

1. Campbell, R. J., ed. 1992. A Guide to Mangos in Florida. Fairchild Tropical Garden, Miami.

2. Capote, M., Lima, H., Cabrera, A. M., Naveira, A., and Blanco, Y. M. 1989. Agrupacion fenotipica de 31 cultivares de mango sobre la base de variables morfologicas y de resistencia a las enfermedades. Ciencia Tec. Agric., Cuba. 2(4):119-127.

3. Ellingboe, A. H. 1976. Genetics of host-parasite interactions. Pages 761-778 in: Physiological Plant Pathology. R. Heitefuss and P. H. Williams, eds. Springer-Verlag, Berlin.

4. Gilbert, G. S., and De Steven, D. 1996. Canker disease of seedlings and saplings of Tetragastris panamensis (Burseraceae) caused by Botryosphaeria dothidea in a lowland tropical forest. Plant Dis. 80:684-687.

5. Gongolly, S. R., Singh, R., Katyal, S. L., and Singh, D. 1957. The Mango. New Delhi Indian Council of Agricultural Research. pp. 23-35.

6. Grossenbacher, J. G., and Duggar, B. M. 1911. A contribution to the life histoparasitism and biology of Botryosphaeria ribis. N.Y. Agric. Exp. Stn. 18:1-189.

7. Hanlin, R. T. 1990. Illustrated Genera of 
Ascomycetes. American Phytopathological Society, St. Paul, MN.

8. Heath, M. C. 1987. Evolution of plant resistance and susceptibility to fungal invaders Can. J. Plant Pathol. 9:389-397.

9. Kostermans, A. J. G. H., and Bompard, J.-M. 1993. The Mangoes: Their Botany, Nomenclature, Horticulture and Utilization. IBPGR. Academic Press, San Diego.

10. Maheshwari, P., and Sachar, R. C. 1963. Polyembryony. Pages 265-296 in: Recent Advances in the Embryology of Angiosperms. P. Maheshwari, ed. Int. Soc. Plant Morphol., Delhi, India.

11. McSorley, R., Campbell, C. W., and Goldweber, S. 1981. Observations of mango decline in south Florida. Proc. Fla. State Hortic. Soc. 93:132-133.

12. McSorley, R., Parrado, J. L., and Goldweber, S. 1981. Plant-parasitic nematodes associated with mango and relationship to tree condition.
Nematropica 11:1-9.

13. Ploetz, R. C., Benscher, D., Vazquez, A., Colls, A., Nagel, J., and Schaffer, B. 1996. A reexamination of mango decline in Florida. Plant Dis. 80:664-668.

14. Popenoe, W. 1920. Manual of Tropical and Subtropical Fruits. McMillan, New York.

15. Ramos, L. J., Lara, S. P., McMillan, R. T., Jr., and Narayanan, K. R. 1991. Tip dieback of mango (Mangifera indica) caused by $\mathrm{Bo}$ tryosphaeria ribis. Plant Dis. 75:315-318.

16. Rhodes, A. M., Campbell, C., Malo, S. E., and Carmer, S. G. 1970. A numerical taxonomic study of the mango (Mangifera indica L.). J. Am. Soc. Hortic. Sci. 95:252-256.

17. Sachar, R. C., and Chopra, R. N. 1957. A study of endosperm and embryo in Mangifera L. Indian J. Agric. Sci. 27:219-228.

18. Schaffer, B. 1994. Decline. Page 43 in: Compendium of Tropical Fruit Diseases. R. C. Ploetz, G. A. Zentmyer, W. T. Nishijima, K.
G. Rohrbach, and H. D. Ohr, eds. American Phytopathological Society, St. Paul, MN.

19. Schaffer, B., Larson, K. D., Snyder, G. H., and Sanchez, C. A. 1988. Identification of mineral deficiencies associated with mango decline by DRIS. HortScience 23:617-619.

20. Schnell, R. J., and Knight, R. J., Jr. 1993 Genetic relationships among Mangifera spp. based on RAPD markers. Mango IV Symposium. Acta Hortic. 341:86-92.

21. Smith, P. F., and Scudder, K., Jr. 1951. Some studies of mineral deficiency symptoms in mango. Proc. Fla. State Hortic. Soc. 64:243 248.

22. Stevens, N. E., and Shears, C. L. 1929. Bo tryosphaeria and Physalospora in Hawaiian Islands. Mycologia 21:313-320.

23. Sturrock, D. 1944. Notes of the mango. Atkins Institute of the Arnold Arboretum of the University of Harvard. Soledad Garden. Soledad, Cienfuegos, Cuba. 